\title{
Interleaver Design Methods for Turbo Codes
}

\author{
Kenneth S. Andrews ${ }^{1}$ \\ Cornell University \\ Ithaca, NY 14843 USA \\ Email andrews@ee.cornell.edu
}

\author{
Chris Heegard \\ Cornell University \\ Ithaca, NY 14843 USA \\ Email heegard@ee.cornell.edu
}

\author{
Dexter Kozen \\ Cornell University \\ Ithaca, NY 14843 USA \\ Email kozen@cs.cornell.edu
}

\begin{abstract}
It is generally assumed that when a turbo decoder is operating at low bit error rates, error sequences have small Hamming weights. From this, and properties of turbo encoders, a mathematical structure is developed for interleaver design, permitting the identification of quantitatively optimal interleavers. Simulations show the math captures some but not all the essential characteristics of a successful interleaver. Modifying a random interleaver according to the mathematical ideas gives excellent simulation results.
\end{abstract}

\section{INTRODUCTION}

The function of the interleaver in the standard rate $1 / 2$ turbo encoder presented in [1] is to assure that at least one of the three codeword components (one systematic and two punctured Binary Recursive Filter (BRF) outputs) has high Hamming weight. As shown in [2], weight-two input sequences dominate in the "average" turbo code distance spectrum, which motivates using primitive BRF feedback polynomials. For a better turbo code, we can design an interleaver of permutation length $p$ that maximizes the minimum Hamming weight generated by weight-two inputs. This requires maximizing:

$$
\sigma_{\pi, \nu} \equiv \min _{i, j}|j-i|+|\pi(j)-\pi(i)|
$$

where the min is over $1 \leq i, j \leq p$ such that,

$$
(j-i) \bmod \left(2^{\nu}-1\right)=(\pi(j)-\pi(i)) \bmod \left(2^{\nu}-1\right)=0,
$$

where $\pi$ is the interleaver function and $\nu$ is the constraint length of the BRFs. A similar problem, more prominent in the literature, replaces the sum in (1) with the maximum:

$$
s_{\pi, \nu} \equiv \min _{i, j}|j-i| \vee|\pi(j)-\pi(i)| \quad 1 \leq i, j \leq p
$$

An alternate method for interleaver design is to disperse symbols as widely as possible. Thus, inputs generating short bursts of activity via one BRF will be dispersed and generate long bursts via the other BRF. Two natural definitions of this dispersion are exactly (1) and (3), without restriction (2).

\section{Solutions and Simulation Results}

When $\sigma$ is odd and $p=\left(\sigma^{2}-1\right) / 2$, there are exactly two solutions to (1):

$$
\pi(i)=(\sigma i+\sigma-1) \bmod \frac{\sigma^{2}+1}{2} \quad 0 \leq i \leq \frac{\sigma^{2}-1}{2}
$$

and its inverse. Similar solutions exist for even $\sigma$, and for the other problems posed.

Computer simulations of interleavers derived using (2) with either (1) or (3) perform badly, because they do not disperse

${ }^{1}$ This work was supported by NSF grants NCR-9520981 and CCR-9708915.

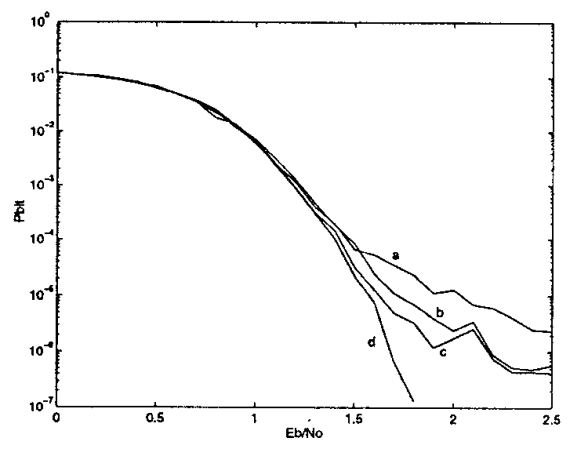

activity from input sequences of weight three and higher. Simulations from interleavers derived without (2) perform better. Using (4) with $\sigma=49, p=1200$ and BRF transfer function $\left(1+D+D^{2}+D^{3}\right) /\left(1+D^{2}+D^{3}\right)$ gives curve $a$ in the figure after 20 iterations.

\section{ADDING RANDOMNESS}

Curve $c$ shows simulation results from a pseudo-random interleaver of length 1176 with the same BRFs. The regularity of the mathematically derived interleavers permits "rectangular" error events with high multiplicities as described in [1], among others; the irregularity of the pseudo-random interleaver virtually eliminates them.

By culling out pairs analytically identified as weak from a pseudo-random interleaver, we can gain the advantages of the analysis and of the irregularity. One effective method is to choose $s_{1}$ and $s_{2}$ and generate $\pi$ one point at a time. For each $i \in[1, p]$ taken sequentially, random values are considered for $\pi(i)$ until one is found satisfying (3) for $s_{\pi}=s_{1}$, and both (3),(2) for $s_{\pi, \nu}=s_{2}$. When no satisfactory values remain, the unused ordinate values are inserted in the unfinished interleaver such that (3),(2) are not violated. While this algorithm may not complete successfully for large $s_{1}$, results are satisfactory. Simulations using such an interleaver with $p=1176, s_{1}=23, s_{2}=49$ and the same BRFs (curve $d$ ) outperform other cases considered here, including an interleaver constructed according to [3] (curve $b$ ). Virtually all remaining errors observed are due to non-convergence of the iterative decoder.

\section{REFERENCES}

[1] C. Berrou and A. Glavieux, "Near Optimum Error Correcting Coding And Decoding: Turbo-Codes," IEEE Trans. Comm., vol. COM-44, no. 10, pp. 1261-1271, Oct 1996.

[2] Perez, Seghers, Costello, "A Distance Spectrum Interpretation of Turbo Codes," IEEE Trans. Inform. Theory, vol. 42, No. 6 pp. 1698-1709, Nov. 1996.

[3] Podemski, Holubowicz, Berrou, Battail, "Hamming Distance Spectra of Turbo-Codes," Annales Telecommunication, vol. 50, no. 9, pp. 790-797, Oct. 1995. 